\title{
Balance and visual reliance in post-COVID syndrome patients assessed with a robotic system: a multi-sensory integration deficit
}

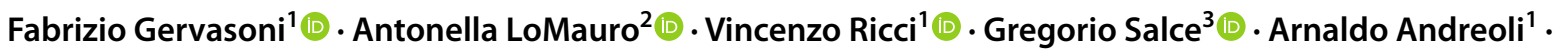 \\ Alessandro Visconti ${ }^{4} \cdot$ Leonardo Pantoni ${ }^{5}$ (i)
}

Received: 6 September 2021 / Accepted: 29 September 2021 / Published online: 6 October 2021

(c) Fondazione Società Italiana di Neurologia 2021

\begin{abstract}
The symptoms of SARS-CoV-2 infection are not limited to the acute phase, with vertigo, peripheral neuropathies, headache, fatigue, memory loss, and depression being the most common post-acute clinical manifestations. Such post-COVID syndrome is a new clinically relevant challenge for diagnosis and therapy. Our goal was to quantify deficit in balance and proprioception related to post-COVID syndrome and, in this sense, we prospectively analyzed data of 66 post-COVID-19 outpatients (mean age 47.3 \pm 11.1 years, 50 females, 25 hospitalized), evaluated using the robotic device hunova. The dynamic balance was assessed with open (OE) and closed eyes (CE) and three indexes, proportional to subject instability, were measured: the sway path and two oscillation ranges. Hospitalized group showed the worst performance with respect to non-hospitalized patients and normality range in both visual conditions for the sway path and the oscillation ranges, with the worst performance being with CE. When compared to normality ranges, post-COVID patients were significantly more distant from normality in the $\mathrm{OE}$ condition compared to the $\mathrm{CE}$ condition. These results suggest that independently from the severity of the disease experienced, post-COVID syndrome makes the elastic balance test performances more distant from the normality when the subject integrates vision, somatosensory information, and vestibular information. In the absence of visual feedback, patients seem to implement compensatory strategies, presumably seeking more significant feedback from the lower limbs, which improve their performance. These data suggest a new mechanism of the post-COVID syndrome that deserves further investigation for its potential impact on activities of daily living.
\end{abstract}

Keywords SARS-CoV-2 infection · Robotic system · Multi-sensory integration deficit · Balance $\cdot$ Post-COVID syndrome

The symptoms of SARS-CoV-2 infection are not limited to the acute phase and may persist many months after the testing becomes negative [1], also after the milder form of the disease [2]. These manifestations affect not only the lungs - primary organs involved during the first stages—but

Fabrizio Gervasoni

fabrizio.gervasoni@ fisiatramilano.it

1 Physical and Rehabilitation Medicine Unit, Luigi Sacco University Hospital, Asst Fatebenefratelli Sacco, Via G.B. Grassi, 74, 20157 Milan, Italy

2 Dipartimento di Elettronica, Informazione e Bioingegneria, Politecnico Di Milano, Milan, Italy

3 Specialisation School Physical and Rehabilitation Medicine, University of Milan, Milan, Italy

4 Direzione Generale, Asst Fatebenefratelli Sacco, Milan, Italy

5 Luigi Sacco, Department of Biomedical and Clinical Sciences, University of Milan, Milan, Italy also other organs, apparatus, and systems as the central and peripheral nervous systems. Among the most common clinical manifestations are vertigo, myalgias, peripheral neuropathies, headache, sleep disturbances, brain fog, fatigue, memory loss, and depression.

Considering neuromotor COVID-19 sequelae, two are the possible etiopathogenetic mechanisms: (1) immobilization and body pronation in patients admitted to intensive care unit and (2) SARS-CoV-2 neurotropism [3]. The former could cause joint stiffness and compression neuropathies [4]; the latter could be related to peripheral neuropathies, balance disorders, and diffuse myalgias.

The post-COVID syndrome is becoming a new clinically relevant challenge that needs profound attention from the therapeutic point of view, but also in terms of diagnosis. Building rational and effective strategies for treating the disabling consequences of COVID-19 is a clinical priority, with important economic implications and relevance 
for public health. Only with a better knowledge of postCOVID syndrome and standardized clinical and instrumental screening protocols, it will be possible to identify signs and symptoms of COVID-19 multi-systemic involvement at an early stage and promptly set up an appropriate and effective rehabilitation treatment.

In this sense, we prospectively analyzed the data of post-COVID-19 outpatients at the Rehabilitation Unit of the Luigi Sacco Hospital (Milan, Italy) diagnosed over the last 6 months. Sixty-six patients (mean age $47.3 \pm 11.1$ years, age range $22-65,50$ females) were evaluated (inclusion criteria: positive test for COVID-19 in the last 12 months and negative test for COVID-19 at the time of assessment) and treated according to the national guidelines and to the ethical standards of the Declaration of Helsinki. They all signed informed consent.

The severity of COVID-19 was scored from 1 to 6 ( 1 =asymptomatic; 2 = paucisymptomatic illness, not requiring hospitalization; $3=$ hospitalization without the need for oxygen therapy; $4=$ hospitalization with need for oxygen therapy; $5=$ hospitalization with need for ventilatory support with CPAP helmet; and $6=$ hospitalization with need for oro-tracheal intubation). Accordingly, 25 patients were classified as hospitalized ( $\mathrm{H}$, score $\geq 3)$, and 41 as not hospitalized $(\mathrm{NH}$, score $\leq 2)$.

Our main goal was to quantify - with a standardized and accurate protocol - the deficit in balance and proprioception in patients with a diagnosis of post-COVID syndrome, using the innovative robotic device hunova [5]. The technologic device integrates robotic platforms and wearable devices to generate complex and variable interactions with the patient for functional sensory motor evaluation and rehabilitation treatment of the ankles, trunk, and lower limbs. The objective measurement of the aforementioned interactions, combined with dedicated elaborations of the data collected, allows planning a tailored rehabilitation treatment, different for each and every patient.

In the present study, the dynamic balance was evaluated with open (OE) and closed eyes (CE). Patients had to maintain balance while standing on a platform with a slight instability that follows the oscillations of their bodies. Three indexes, proportional to subject instability, were measured: the sway path and the oscillation ranges in the anteroposterior (AP) and medio-lateral (ML) directions (Fig. 1) [6-8]. Finally, for each visual condition, and each subject, an overall and normalized score of balance performance was computed. This was a mean $z$-score that compared the

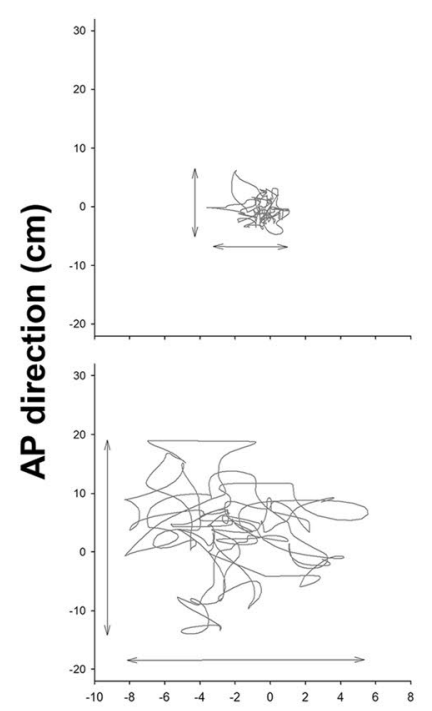

$M L$ direction $(\mathrm{cm})$
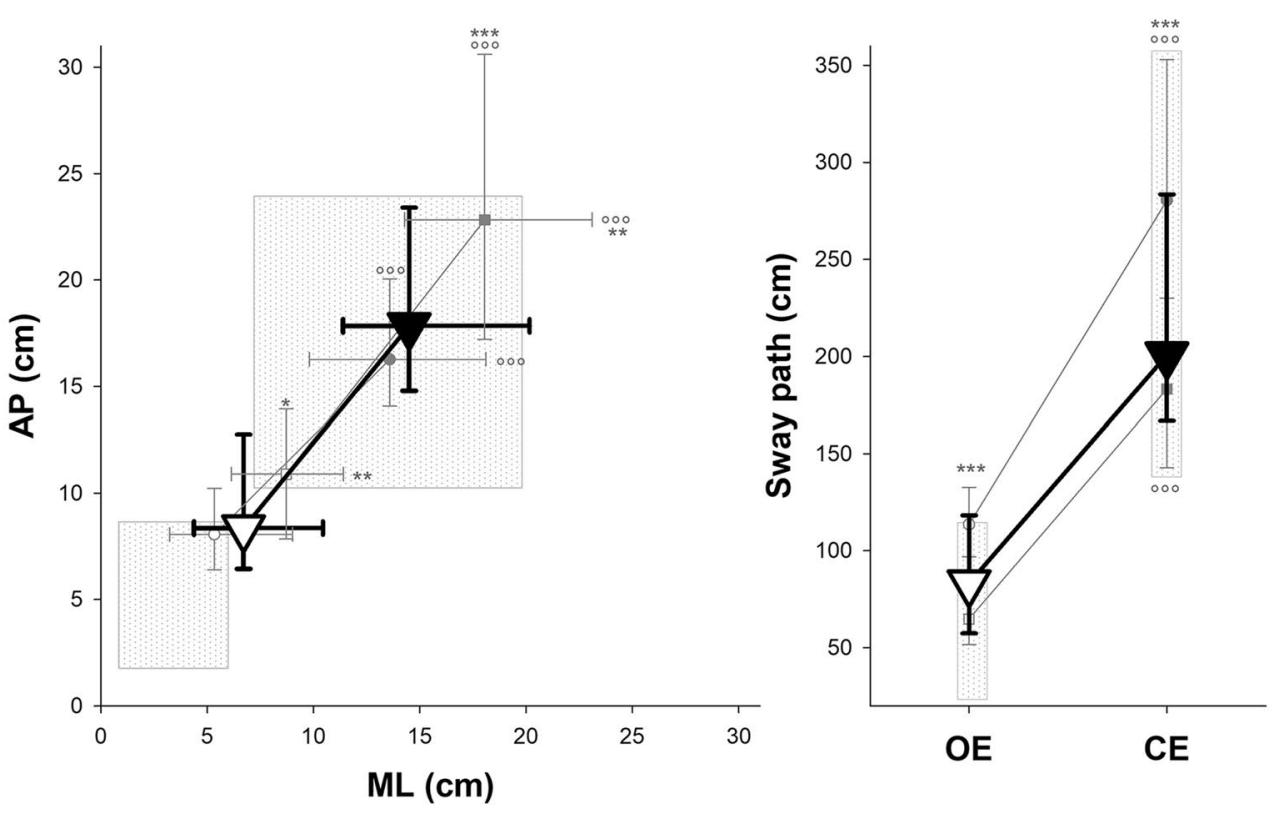

Fig. 1 Left panels: Representative of the center-of-foot pressure trace during the dynamic balance of a patient with open (top) and closed (bottom) eyes. The oscillation ranges in the antero-posterior (AP) and medio-lateral (ML) directions were quantified by projecting the angular displacement of the platform on a plane. The sway path is the length of the trajectory. Middle panel: Median (symbols) and interquartile range (whiskers) of the ML ( $x$-axis) and AP ( $y$-axis) of overall post-COVID syndrome patients (triangles), also splint in hospitalized (circles) and non-hospitalized (squares) patients, with open eyes (OE, open symbols) and closed eyes (CE, close symbols). $*, * * *: p<0.05,0.001$ hospitalized vs non-hospitalized patients; ${ }^{\circ}$ : $p<0.001 \mathrm{CE}$ vs OE. Right panel: Median (symbols) and interquartile range (whiskers) of the sway path of overall post-COVID syndrome patients (triangles), also splint in hospitalized (circles) and non-hospitalized (squares) patients, with open eyes (OE, open symbols) and closed eyes (CE, close symbols). ***: $p<0.001$ hospitalized vs nonhospitalized patients; ${ }^{\circ \circ}: p<0.001 \mathrm{CE}$ vs $\mathrm{OE}$ 
values of the single subject with the corresponding mean and standard deviation of normality, assessed as a sample of 80 healthy young subjects (mean age $26.4 \pm 5.9$ years, 40 females) and 41 older healthy subjects (mean age $50.7 \pm 7.4$ years, 20 females) acquired with the same protocol.

Because normality distribution failed (Kolmogorov-Smirnov tested), the Wilcoxon signed-rank test was applied to compare the two visual conditions (OE vs CE) while the Mann-Whitney $U$ test to analyze group differences (H vs NH patients). The Bonferroni correction for multiple comparisons was performed, with the significance threshold set at 0.025 (MATLAB 2017b; MathWorks, Natick, MA, USA).

The $\mathrm{H}$ group showed the worst performance with respect to NH patients and normality range. This occurred in both visual conditions for the sway path and the oscillation ranges. As expected, the worst performance was with CE in both groups (Fig. 1). When compared to normality ranges, post-COVID patients were significantly more distant from normality in the condition with $\mathrm{OE}$ compared to the $\mathrm{CE}$ condition (Fig. 2).

These results showed that, independently from the severity of the disease experienced, post-COVID syndrome makes the performances in the elastic balance test achieved with $\mathrm{OE}$ be more distant from the normality than the same test performed with $\mathrm{CE}$. These unexpected results led us to some considerations. In the OE condition, the subject integrates the information of three sensory systems-vision, somatosensory, and vestibular information-while visual feedback is missing with CE. Once the visual component is excluded, patients with post-COVID syndrome seemed to improve their performance presumably because they seek more significant feedback from the lower limbs. The unstable platform returns less accurate sensory data, therefore requiring greater adaptation and more precise use of somatosensory information. In the absence of visual feedback, patients seem to implement compensatory strategies that improve their performance. A similar failure in integrating different sensory inputs (visual and somatosensory) was observed in older adults and also in multiple sclerosis patients whose visual reliance prevails when feedback from lower limbs is compromised [9].

We can speculate that the neurotropism, the neuroinflammatory component of the COVID-19 infection, and the myopathic changes induced by the SARS-CoV-2 infection might be the causes contributing to this multi-sensory integration deficit, although, emotional, psychological, or psychiatric aspects might also play a role. A functional neurological disorder cannot be excluded [10].

These data suggest a new mechanism of the post-COVID syndrome that deserves further investigation for its potential impact on activities of daily living.

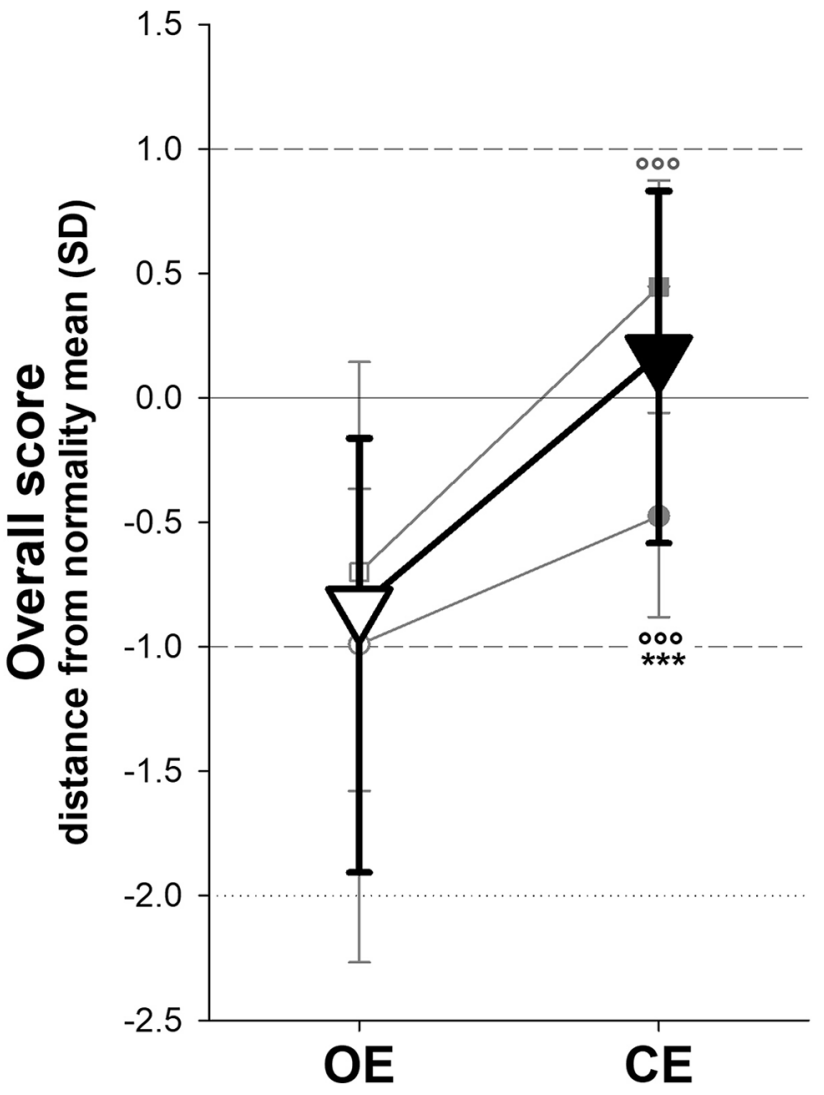

Fig. 2 Median (symbols) and interquartile range (whiskers) of the overall score compared to normality (zero line) of overall postCOVID syndrome patients (triangles), also splint in hospitalized (circles) and non-hospitalized (squares) patients, with open eyes (OE, open symbols) and closed eyes (CE, close symbols). ***: $p<0.001$ hospitalized vs non-hospitalized patients; ${ }^{\circ 0}: p<0.001 \mathrm{CE}$ vs $\mathrm{OE}$

Data availability Data available on request due to privacy/ethical restrictions.

Code availability Not applicable.

\section{Declarations}

Ethical approval The study was registered by the local ethical committee of Ospedale Luigi Sacco, Polo Universitario, ASST Fatebenefratelli Sacco (Comitato Etico Area I: 209/2021).

Consent to participate and for publication All patients signed a written informed consent form to participate to the study and to have their date used for publication.

Conflict of interest None. 


\section{References}

1. Carfî A, Bernabei R, Landi F for the Gemelli Against COVID19 Post-Acute Care Study Group (2020) Persistent symptoms in patients after acute COVID-19. JAMA 324(6):603-605. https:// doi.org/10.1001/jama.2020.12603.

2. Augustin M, Schommers P, Stecher M et al (2021) Post-COVID syndrome in non-hospitalized patients with COVID-19: a longitudinal prospective cohort study. Lancet 6(July 2021):100122. https://doi.org/10.1016/j.lanepe.2021.100122

3. Hu J, Jolkkonen J, Zhao C (2020) Neurotropism of SARS-CoV-2 and its neuropathological alterations: Similarities with other coronaviruses. Neurosci Biobehav Rev 119:184-193. https://doi.org/ 10.1016/j.neubiorev.2020.10.012

4. Fernandez CE, Franz CK, Ko JH, Walter JM, Koralnik IJ et al (2021) Imaging review of peripheral nerve injuries in patients with COVID-19. Radiology 298(3). https://doi.org/10.1148/ radiol.2020203116

5. Saglia JA, De Luca A, Squeri V, Ciaccia L, Sanfilippo C, Ungaro S, De Michieli L (2019) Design and development of a novel core, balance and lower limb rehabilitation robot: hunova, IEEE 16th International Conference on Rehabilitation Robotics (ICORR), Toronto, Canada, June 24-28, 2019

6. Cella A, De Luca A, Squeri V, Parodi S, Vallone F, Giorgeschi A, Senesi B, Zigoura E, Guerrero KLQ, Siri G, De Michieli L, Saglia J, Sanfilippo C, Pilotto A (2020) Development and validation of a robotic multifactorial fall-risk predictive model: a one-year prospective study in community-dwelling older adults. PlosOne 15(6):e0234904. https://doi.org/10.1371/journal.pone.0234904. eCollection
7. De Luca A, Squeri V, Barone L, Vernetti H, Ricci S, Pisu I, Cassiano C, Capra C, Lentino C, De Michieli L, Sanfilippo C, Saglia J (2020) Checchia GA (2020) Dynamic stability and trunk control improvements following robotic balance and core stability training in chronic stroke survivors: a pilot study. Front Neurol 11:494. https://doi.org/10.3389/fneur.2020.00494.eCollection

8. Cella A, De Luca A, Squeri V, Parodi S, Puntoni M, Vallone F, Giorgeschi A, Garofalo V, Zigoura E, Senesi B, De Michieli L, Saglia JA, Sanfilippo C, Pilotto A (2020) Robotic balance assessment in community-dwelling older people with different grades of impairment of physical performance. Aging Clin Exp Res 32(3):491-503. https://doi.org/10.1007/s40520-019-01395-0

9. Yeh TT, Cluff T, Balasubramaniam R (2014) Visual reliance for balance control in older adults persists when visual information is disrupted by artificial feedback delays. PLoS ONE 9(3):e91554. https://doi.org/10.1371/journal.pone.0091554.eCollection

10. Espay AJ, Aybek S, Carson A, Edwards MJ, Goldstein LH, Hallett M, LaFaver K, LaFrance WC Jr, Lang AE, Nicholson T, Nielsen G, Reuber M, Voon V, Stone J, Morgante F (2018) Current concepts in diagnosis and treatment of functional neurological disorders. JAMA Neurol 75(9):1132-1141. https://doi.org/10.1001/ jamaneurol.2018.1264

Publisher's note Springer Nature remains neutral with regard to jurisdictional claims in published maps and institutional affiliations. 